\title{
Les systèmes d'approvisionnement en semences de mils et sorghos au Mali : production, diffusion et conservation des variétés en milieu paysan
}

\author{
Harouna Coulibaly ${ }^{1}$ \\ Didier Bazile ${ }^{2}$ \\ Amadou Sidibé ${ }^{1}$ \\ Géraldine Abrami ${ }^{3}$ \\ ${ }^{1}$ Institut d'économie rurale (IER), \\ Sotuba, \\ BP 262, \\ Bamako \\ Mali \\ <hscoulibaly@yahoo.fr> \\ <sidibea20@yahoo.fr>
}

${ }^{2}$ Centre de coopération internationale en recherche agronomique pour le développement (Cirad),

Département Environnements \& Sociétés, UPR 47 " GREEN ",

Campus international de Baillarguet,

TA C-47/F,

34398 Montpellier cedex 5

<didier.bazile@cirad.fr>

${ }^{3}$ Centre de coopération internationale en recherche agronomique pour le développement (Cirad),

Institut de recherche pour l'ingénierie de l'agriculture et de l'environnement (Cemagref),

UMR G-Eau,

361, rue Jean-François Breton,

BP 5095

34398 Montpellier

<geraldine.abrami@cemagref.fr>

\begin{abstract}
Résumé
Le Mali, pays sahélien, est situé dans un centre de diversité d'espèces parmi lesquelles figurent les mils et sorghos. Ces deux espèces constituent la base de l'alimentation des populations rurales. La reproduction à la ferme représente la principale source d'approvisionnement en semences du paysan. Diverses raisons - sécheresse, ou mauvaise production - font que les agriculteurs sont en permanence à la recherche de nouvelles variétés plus performantes. Le système semencier paysan (SSP), informel, est le principal fournisseur de semences pour le paysan, loin devant le système semencier formel (SSF), étatique. Cette note de recherche présente le cadre de diffusion des variétés améliorées du SSF et le compare avec les mécanismes d'échanges de semences entre paysans dans le cadre du SSP. La discussion porte sur la capacité des deux systèmes à assurer, d'une part, la diffusion de matériel génétique adapté aux besoins des paysans, et, d'autre part, la conservation des variétés traditionnelles. L'émergence d'organisations paysannes (OP) souhaitant jouer un rôle actif dans la multiplication des semences certifiées pour améliorer l'approvisionnement de leurs membres en matériel amélioré pose la question de l'impact de ce rôle d'interface entre ces deux systèmes semenciers. L'analyse s'appuie sur différents rapports et interviews des responsables des antennes régionales du SSF et divers travaux de Bazile et al. sur le SSP. L'utilisation de diagrammes en langage UML (Unified Modeling Language) permet de formaliser un modèle conceptuel des deux systèmes avec leurs interactions. En général, aucun de ces systèmes ne couvre efficacement et complètement les besoins des paysans en semences de mil et sorgho.
\end{abstract}

Mots clés : Mali ; mil ; sorgho ; système semencier ; organisation paysanne ; modélisation.

Thèmes : économie et développement rural ; productions végétales.

\section{Abstract}

Seed supply systems of pearl millet and sorghum in Mali: Production, diffusion and conservation of varieties

Mali is a Sahelian country located in a center of great species diversity among which are listed millet and sorghum. These two species are the main staple food crops. On-farm production represents the main source of seeds for peasants. Various reasons such as drought or bad production motivate farmers to look permanently for more productive varieties. The peasants' seed system (PSS) which is informally organized is the principal source of seed supply for peasants and more important than the formal seed system (FSS). The research note presents the framework of diffusion of improved varieties in the FSS and compares it with the seed exchange mechanisms between peasants within the PSS framework. The discussion is related to the capacity of the two systems to ensure on the one hand the diffusion of genetic material adapted to the needs of peasants and on the other hand the conservation of local varieties. The emergence of Farmers' Organizations (FO), who want to play a key role in the production of certified seeds to improve the supply of their members with improved material, raises the question of the impact of this role of interface between these two seed systems. The analysis is based on various reports and interviews with people in charge at the regional representations of the FSS and on the work of Bazile et al. on PSS. The use of diagrams in Unified Modeling Language (UML) makes it possible to formalize a conceptual model of the two systems with their interactions. In

Tirés à part : H. Coulibaly 
general, none of these systems satisfies peasants' need of millet and/or sorghum seeds.

Key words: Mali; millets; sorghum grain; seed supply system; farmers organizations; modelling.

Subjects: economy and rural development; vegetal productions.

e Mali, pays sahélien, est situé dans un centre de diversité d'espèces parmi lesquelles figurent les mils (Pennisetum glaucaum) et les sorghos (Sorghum bicolor). Ils constituent la base de l'alimentation pour la majorité de la population rurale. La production à la ferme représente la principale source d'approvisionnement en semences du paysan. Néanmoins, pour des raisons de sécheresse, de ravageurs ou de mauvaise production, le paysan peut manquer de semences de qualité ou désirer modifier ses choix variétaux. Il peut alors avoir recours à deux systèmes: le système semencier formel (SSF) et le système semencier paysan (SSP) qui coexistent sur le même espace (Almekinders et Louwaars, 2002). Depuis 2002 nous assistons à l'émergence d'autres acteurs, tels les organisations paysannes (OP) ou les organisations non gouvernementales (ONG), qui participent activement à la couverture des besoins en semences des producteurs et remettent en question cette dichotomie entre les deux systèmes semenciers.

\section{Matériel et méthode}

Cette étude détaille la structure et le fonctionnement du Service semencier national (SSN) pour les deux espèces, mil et sorgho. Elle s'appuie sur une analyse de divers rapports d'activités des antennes régionales du SSN complétée par des interviews auprès de responsables de ce service et des paysans. Les données recueillies sont confrontées aux résultats des essais conduits par l'organisation fầtière des OP (Association des organisations professionnelles paysannes, AOPP) dans la région de Ségou sur la production et la diffusion de semences de variétés améliorées. Le fonctionnement du SSP a déjà été décrit dans différents travaux à travers des enquêtes et des ateliers associant les jeux de rôles et la modélisation multiagent (Bazile et al., 2005). Nous utilisons ces connaissances pour expliquer l'articulation des acteurs du SSP avec ceux du SSF (Bazile et al., 2006).
Le langage de modélisation UML (Unified Modeling Language) est utilisé pour représenter les systèmes étudiés en termes d'objets et d'interaction entre ces objets (Booch et al., 2000). Il permet de formaliser un modèle conceptuel reliant le SSF et le SSP, indépendamment d'un langage de programmation. Notre recherche applique ce formalisme à la compréhension de la production et de la diffusion des variétés améliorées de mil et de sorgho au Mali.

\section{Résultats et discussions}

Un système semencier a pour rôle de fournir aux paysans en temps opportun les semences en qualité et quantité suffisantes. Il recouvre à la fois les méthodes de sélection, de production et de diffusion des variétés. Pour répondre à cette mission d'intérêt général, plusieurs services du ministère de l'Agriculture interviennent dans la mise en œuvre de la politique semencière au Mali : l'Institut d'économie rurale (IER), le Système semencier national (SSN), la Direction générale de la réglementation et du contrôle du secteur du développement rural et les structures d'encadrement technique. Le SSN coordonne les activités semencières des services de l'État à l'échelle du pays. II recense les besoins en semences des structures d'encadrement, soumet le programme de production de semences de base à l'IER. Il établit le programme de production des semences et le fait exécuter par des paysans semenciers. Enfin, il finance les activités de contrôle et de certification et assure la commercialisation des semences certifiées (R1 et R2). Les semences de base proviennent d'une multiplication dont l'origine première des semences est la récolte produite par des plantes initiales détenues et conservées par un obtenteur (organisme public ou privé). La première reproduction à partir des semences de base constitue les semences certifiées R1. La seconde repro- duction constitue les semences certifiées R2 issues de R1. Les générations de semences au-delà de la R2 ne sont pas admises à la certification au Mali.

Un protocole d'accord lie le SSN à l'IER pour la fourniture des semences de base. Trois antennes - Samanko, Babougou et M'Pèssoba - participent à la production des semences certifiées R1 de mils et sorghos en collaboration avec les paysans semenciers de villages environnants. Depuis 2005, la production des R2 destinées aux emblavures est réalisée par des coopératives paysannes constituées dans le cadre du Projet d'appui à la filière semencière (Pafisem). Après les opérations de contrôle au champ, d'analyse et de certification des semences qui sont effectuées par les Directions régionales de l'Agriculture et le Laboratoire de semences de Bamako, les R2 sont stockées dans les magasins des antennes et au siège du SSN où elles sont vendues aux demandeurs (figure 1).

Malgré un SSF en apparence opérationnel, on note que les variétés améliorées ont enregistré peu de progression dans les surfaces cultivées ( 5 à $10 \%$ ) à cause, d'une part, de leur inadaptation aux conditions socio-économiques et environnementales locales et, d'autre part, du fait du manque d'information, ou de connexion, entre le paysan et les distributeurs de semences améliorées.

Cela détermine une grande disparité entre l'offre en semences, la demande et les ventes. Pour les mils et sorghos, lors d'une bonne année de production (comme ce fut le cas en 2003-2004), il y a très peu d'achat de semences. Mais en année de mauvaise production (par exemple 2004-2005), l'offre de semences certifiées est souvent insuffisante par rapport aux besoins des paysans. Cela illustre bien la difficulté de maitriser la production de semences pour les mils et les sorghos qui sont des céréales d'autoconsommation dont la reproduction des semences à la ferme constitue la pratique la plus courante. Le fait que les échanges restent gratuits en milieu paysan constitue un handicap de plus pour la 


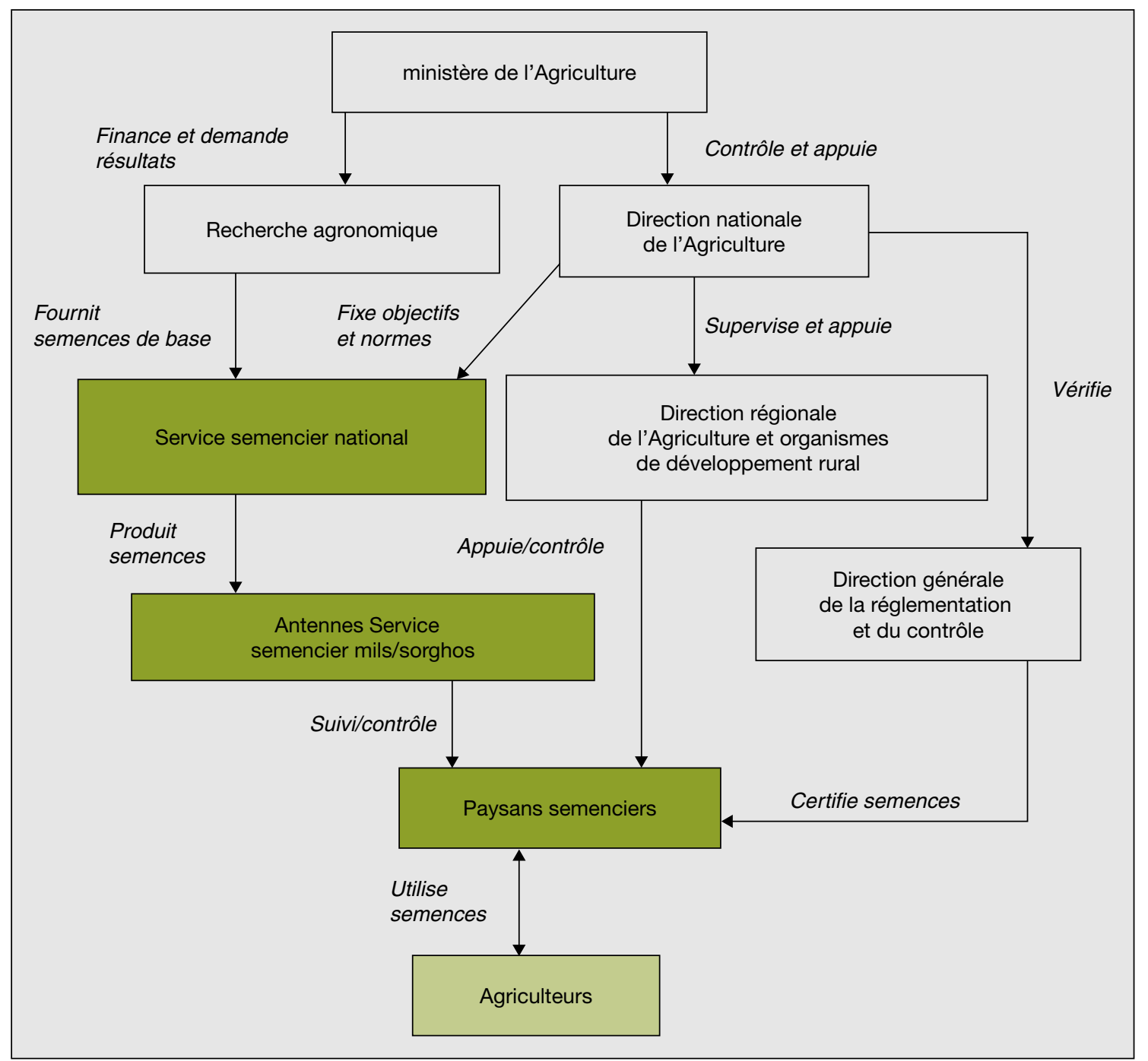

Figure 1. Production et diffusion de semences certifiées par le Système semencier national.

Figure 1. Production and distribution of certified seeds in the formal seed system.

commercialisation des variétés améliorées de ces deux espèces.

C'est pourquoi l'AOPP a mis en place des activités semencières dans la région de Ségou (figure 2) pour répondre au besoin des paysans en termes d'amélioration des variétés sur deux critères : la productivité et la réduction de la longueur du cycle. Dans un premier temps, elle identifie avec les techniciens du SSN quelques variétés adaptées aux zones géographiques où elle souhaite intervenir. Puis, elle recherche les semences de base ou les R1 auprès de la recherche agronomique et quelques $\mathrm{OP}$ produisent les R2 pour les besoins des paysans des localités concernées. L'AOPP prend en charge les frais de certification et diffuse les semences produites par le biais des OP. Ces semences certifiées sont vendues aux adhérents des OP membres ou non de l'AOPP à un prix inférieur à celui du SSF pour en faciliter l'accès à un maximum de paysans.

Dans le SSP, la sélection de semences repose sur les savoirs locaux et les paysans pratiquent une sélection massale à chaque génération pendant les récoltes en fonction d'objectifs de production et une appréciation propre des variétés. Les critères de maintien des variétés locales sont larges et concernent aussi bien le cycle, la stabilité du rendement, le goût, la conservation que la résistance aux maladies. La reproduction des semences chaque année sur le champ paysan conduit à une évolution adaptative des variétés. C'est pourquoi toutes les exploitations peuvent être demandeurs et fournisseurs de semences, selon le moment, pour répondre au devoir de solidarité au sein des communautés (en fonction du lignage ou du statut social). Le fait que certains paysans soient membres d'OP permet d'introduire de nouvelles variétés améliorées dans le village et d'enrichir la diversité génétique existante. Après introduction, la sélection des semences des variétés traditionnelles et améliorées est faite selon les mêmes techniques. Ensuite, la diffusion des variétés améliorées suit les mêmes mécanismes d'échanges que les variétés locales. Ainsi, on voit bien l'importance et le rôle que peuvent jouer les OP dans l'introduction et la circulation du matériel amélioré dans les zones rurales enclavées. Néanmoins, il est important de bien comprendre l'articulation du SSP et du SSF avec les OP à leur interface pour pouvoir maîtriser les effets négatifs de diffusion massive d'une 


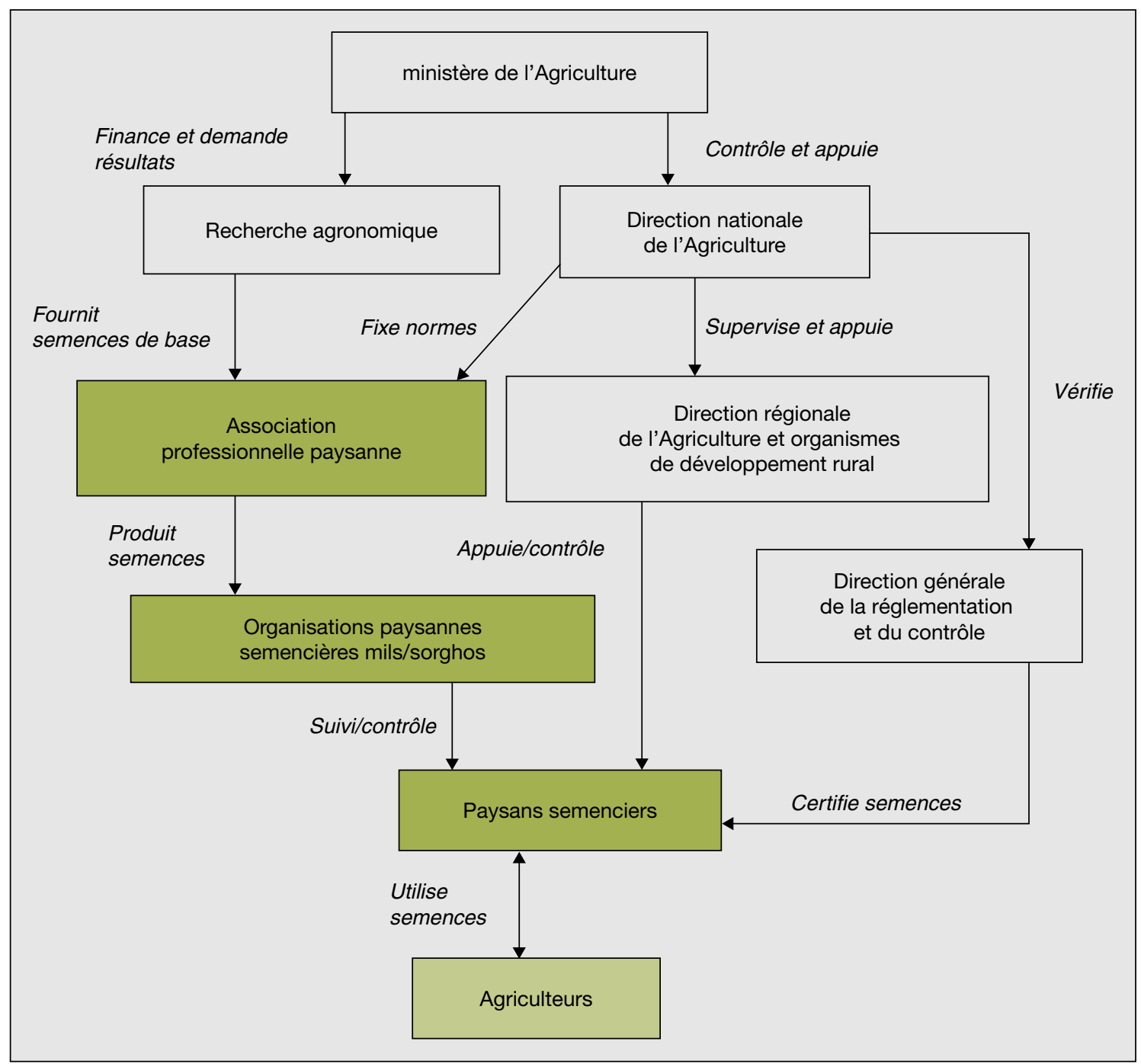

Figure 2. Production et diffusion de semences certifiées par les organisations paysannes.

Figure 2. Production and distribution of certified seeds by farmers' organizations.

seule variété améliorée par un leader paysan reconnu au niveau du village qui contribuerait à accroître l'érosion génétique et la perte de la diversité variétale.

\section{Conclusion}

Le SSN diffuse les variétés modernes suivant un schéma institutionnel centralisé non satisfaisant pour répondre aux besoins spécifiques et non programmés en semences des paysans. Pourtant, même si le SSP assure environ $90 \%$ de la production de semences, il se limite en général à l'aire géographique d'une petite région naturelle et diffuse des semences de qualités assez différentes selon les soins apportés à la sélection par les différents paysans. Depuis 2002, l'AOPP produit et diffuse avec succès des semences améliorées auprès des paysans au Mali. Cette voie nouvelle à l'interface entre le SSP et le SSN devrait permettre d'améliorer l'approvisionnement en semences de qualité pour le paysan.

\section{Références}

Almekinders CJM, Louwaars NP. The importance of the farmers' seed systems in a functional national seed sector. J New Seeds 2002 ; 4 : 15-33.
Bazile D, Abrami G, Dembélé S, Le Page C, Dionnet $\mathrm{M}$, Coulibaly $\mathrm{H}$. Les paysans au cœur de la construction de règles collectives de gestion de I'agrobiodiversité. In : C3ED, ed. Gestion concertée des ressources naturelles et de tion concertee des ressources naturelles et de
l'environnement. Colloque GECOREV, 26-28 juin 2006. St Quentin-en-Yvelines: Institut de recherches pour le développement (IRD); Groupe recherche de recherche et d'échanges technologiques (Gret) ; université de St Quentin, 2006 (cédérom).

Bazile D, Coulibaly $\mathrm{H}$, Soumaré M. Les réseaux sociaux au secours de l'Etat pour l'approvisionnement en semences des paysans. In : Benoit-Cattin M, ed. Les institutions du développement durable des agricultures du Sud. Thème 2. Quelle gestion des ressources et des territoires? Colloque de la Société française d'économie rurale (Sfer), Montpellier, 7-9 novembre 2005. Paris: Sfer, 2005 (cédérom).

Booch G, Rumbaugh J, Jacobson I. Le guide de I'utilisateur UML. Paris : Eyrolles, 2000. 